Full-Length Article

\title{
Music Therapy in Addictions Treatment: A Systematic Review of the Literature and Recommendations for Future Research
}

Kathleen M. Murphy ${ }^{1}$

${ }^{1}$ Loyola University, New Orleans, United States of America

\begin{abstract}
Substance dependence continues to be a major problem in the United States and a treatment approach that is universally successfully has not been identified. This paper will update an earlier review that encouraged the music therapy community to continuing investigating the efficacy of music therapy. 12 studies, met inclusion for this review. The most common music therapy method to be investigated was lyric analysis, followed by music and imagery methods, and then songwriting. The studies reviewed had a moderate to high risk of bias, and were single session posttest only designs conducted on an inpatient detoxification unit. Recommendations include suggestions for reducing bias in future studies and continued research on the myriad music therapy methods that are reportedly used in addiction treatment in the clinical literature.
\end{abstract}

Keywords: music therapy, addictions, substance abuse treatment, systematic review.

multilingual abstract $\mid$ mmd.iammonline.com

Substance dependence continues to be a major problem in the United States. In fact, during 2014, there were 1,614,358 substance abuse treatment admissions for individuals aged 12 and older reported by 50 states, the District of Columbia, and Puerto Rico. $15 \%$ of those individuals had been in treatment 5 or more times previous [1]. Clearly this indicates the need for treatment options that will facilitate recovery and the maintenance of a sober lifestyle. Current treatment includes detoxification, medication assisted treatment, counseling, 12Step Programs, psychosocial therapies and complementary therapists. Music therapy, the use of music experiences designed by a credential music therapist to address health needs, is one such complementary therapy that is being incorporated into addiction treatment programs. A recent survey of 299 treatment facilities found that $14.7 \%$ offered music therapy as part of their treatment program [2].

Systematic reviews, the review of literature related to specific research questions [3] are increasing in number in the published music therapy literature [4]. In fact, Hanson-

\section{PRODUCTION NOTES: Address correspondence to:}

Kathleen M. Murphy, E-mail: kmmurphy@loyno.edu

| COI statement: The author declared that no financial support was given for the writing of this article. The authors have no conflict of interest to declare.
Abromeit \& Moore [5] called for further systematic reviews in order to report findings from the extant literature to inform clinical practice. The first systematic review of music therapy in addictions treatment was conducted by Mays et al. [6]. In their report, they noted a "lack of information and outcomes of the therapy described" (p. 58). Further, they noted that additional research was needed before music therapy could be accepted as a primary treatment modality. This review will update Mays et al. [6] report by reviewing the published music therapy research related to addictions treatment. Specifically, this paper will describe the results of randomized control trials on music based interventions used in addiction treatment including settings for service delivery, music therapy methods used, and outcome measures.

\section{Method}

Three electronic databases (Cumulative Index to Nursing and Allied Health Literature, Medline, and PsycInfo) were used to identify published articles relating to music therapy and addictions treatment that met the following inclusion criteria: 1) available in English; 2) all study participants were adults (18 and older) and were being treated for substance dependence on an inpatient unit; 3) a music therapy protocol implemented by a music therapist was being investigated; 4) the research method met criteria for a randomized control trial as defined 
by the Cochrane Collaborative $[3]^{1}$; and 5) method of randomization was described.

The Preferred Reporting Items for Systematic Reviews and Meta-Analyses (PRISSMA) guidelines [7] were followed in selecting articles for this review. Key words searched in combination with "music therapy" included "alcohol use," "alcohol abuse," "alcohol dependence," "alcoholic;" "drug use," "drug abuse," "drug dependence;" "substance use," "substance abuse," "substance dependence;" "chemical dependence or dependency;" "substance abuse treatment" substance abuse rehabilitation;" "detoxification" and "recovery." This search resulted in an initial list of 117 articles (after duplicates were removed). Abstracts, and then full texts were reviewed. References lists for all relevant articles were reviewed to expand the search. 12 studies met the 5 inclusion criteria (See Figure 1) and were reviewed for this paper. The following data was extracted from each study that met inclusion criteria: demographic information, number of participants, setting, dependent variables and outcome measures, independent variable (music therapy method used), study design, number of sessions; interventionist, and results.

The quality of each study was assessed using the Checklist to Evaluate a Report of Nonpharmacological Trial (CLEARNPT) [8] which was designed to assess standardization, influence of treatment provider, blinding and risk of bias. The CLEAR-NPT has been adapted by music therapists $[4,9]$ to evaluate the quality of music therapy research studies. Following those examples, 1 point was scored if the answer to an item was "yes;" 0 points were scored if the answer was no. A rating of "unclear" was given if there was not enough information in the study to rate the item. The guidelines and ratings used in this review may be found in Table 1 . Scores for each study could range from " 0 " indicating a low quality to "10" indicating high quality. In addition, each study was assessed for risk of bias as described by McKinney \& Honig [4] and Yinger and Gooding [9]. Studies that scored 1 point for questions $1,2,6,7$, and 8 on the quality assessment checklist (Table 2) were considered to have a low risk of bias. Those that scored 1 point on questions 1, 2, 8 and either 6 or 7 , were considered to have a moderate risk of bias. Studies that met scored " 4 " or lower were considered to have a high risk of bias.
Figure 1: Study Selection Flow Chart.

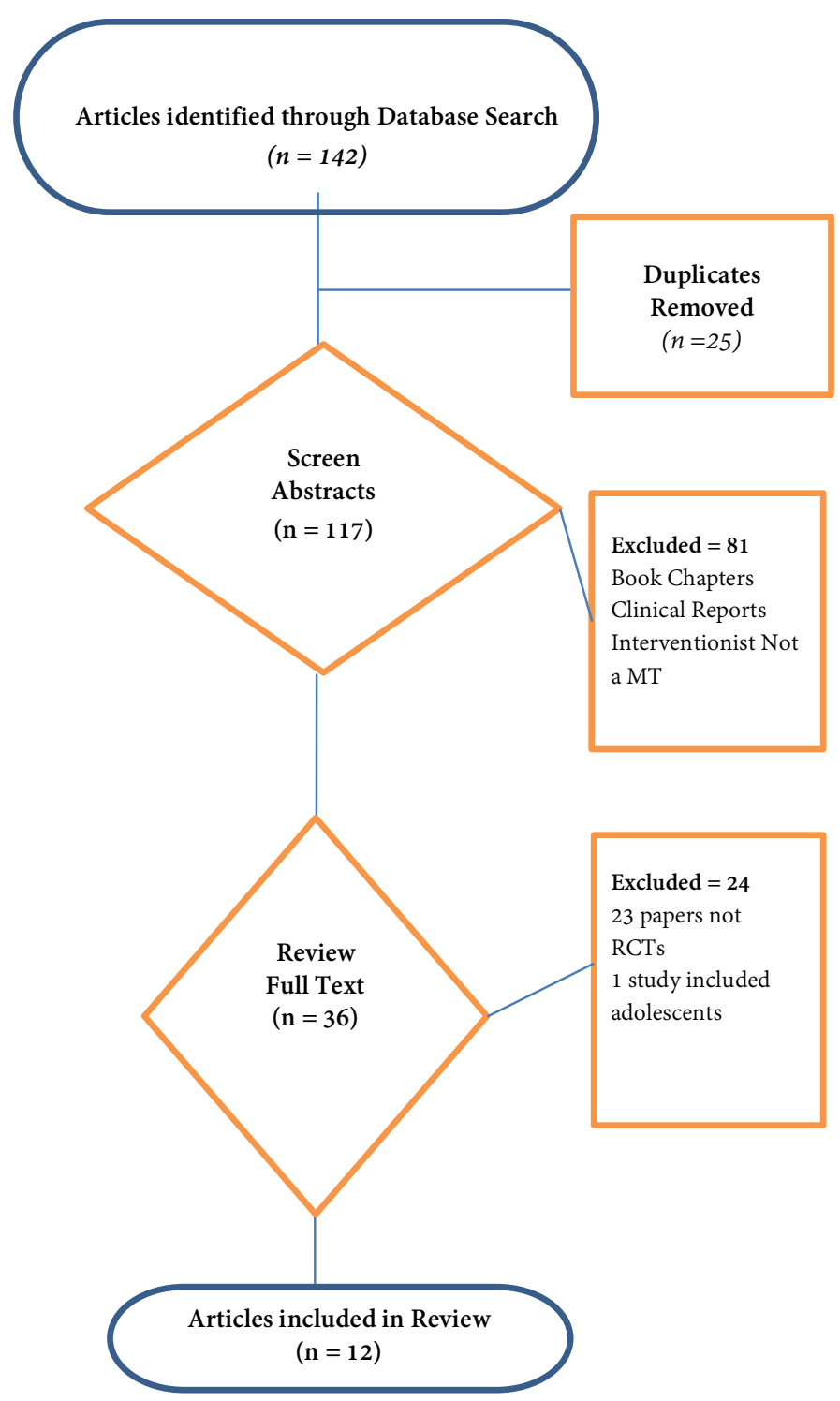

${ }^{1}$ Randomized control trials must meet the following criteria: "the individuals (or other unit) followed in the trial were definitely or possibly assigned prospectively to one of 2 (or more) alternative forms of health care using random allocation or some quasi-random method of allocation (such as alternation, date of birth, or case record number)" [3] 
Table 1: Quality Assessment Rating Items \& Guidelines, adapted from the CLEAR-NPT [8]

1. Was the generation of allocation sequences adequate? Give 1 point only if a suitable method was used to generate the sequence of randomization (i.e. simple randomization via table of random numbers or computer-generated random numbers).

2. Was the treatment allocation concealed? Give 1 point only if participants and investigators enrolling participants could not foresee upcoming group assignments.

3. Were details of the intervention administered to each group made available? Give 1 point only if all of the following information was described in the report or made available in some type of addendum: information about who selected music, type of music; music delivery method; intervention materials, intervention strategies

4. Was care providers' experience or skill in each arm appropriate? Score 1 if the intervention was delivered by a board-certified music therapist with appropriate education and experience.

5. Was participant adherence assessed quantitatively? Score 1 if the number of sessions attended was reported.

6. Were participants adequately blinded? Blinding is not feasible in music therapy studies, assign 0 and rate the sub items:

6a. Were all other treatments and care the same in each randomized group? Score 0.5 if all other treatments and care were the same in each group.

6b. Were non-completers the same in each randomized group? Score 0.5 if non-completers were the same in each group.

7. Were care providers adequately blinded? Blinding is not feasible in music therapy studies, assign 0 and rate the sub items:

7a. Were all other treatments and care the same in each randomized group? Score 0.5 if all other treatments and care were the same in each group.

7b. Were non-completers the same in each randomized group? Score 0.5 if non-completers were the same in each group.

8. Were outcome assessors adequately blinded to assess the primary outcomes? Score 1 for participant-reported outcomes in which the participant is the outcome assessor; Score 0 if blinding is not feasible

8a. If outcome assessors were not adequately blinded, were specific methods use to avoid ascertainment bias (systematic differences in outcome assessment).

9. Was the follow-up schedule the same in each group? Score 1 if planned follow-up schedules were the same in each group.

10. Were the main outcomes analyzed according to the intentionto-treat principle? Score 1 if all participants randomized were included in the analysis and kept in their original group; Score 0 if all participants randomized were not included in the analysis or not kept in their original group. 
Table 2: Quality Assessment

\begin{tabular}{|c|c|c|c|c|c|c|c|c|c|c|c|c|}
\hline \multirow[b]{2}{*}{ Criteria } & \multirow[b]{2}{*}{$\begin{array}{l}\text { Hammer } \\
(1996)\end{array}$} & \multicolumn{11}{|c|}{ Authors } \\
\hline & & $\begin{array}{l}\text { Heiderscheit } \\
(2006)\end{array}$ & $\begin{array}{l}\text { Murphy } \\
(2009)\end{array}$ & $\begin{array}{l}\text { Silverman } \\
(2009)\end{array}$ & $\begin{array}{l}\text { Silverman } \\
(2010)\end{array}$ & $\begin{array}{l}\text { Silverman } \\
\text { (2011a) }\end{array}$ & $\begin{array}{l}\text { Silverman } \\
\text { (2011b) }\end{array}$ & $\begin{array}{l}\text { Silverman } \\
(2012)\end{array}$ & $\begin{array}{l}\text { Silverman } \\
(2014)\end{array}$ & $\begin{array}{l}\text { Silverman } \\
(2015)\end{array}$ & $\begin{array}{l}\text { Silverman } \\
\text { (2016a) }\end{array}$ & $\begin{array}{l}\text { Silverman } \\
\text { (2016b) }\end{array}$ \\
\hline \multicolumn{13}{|l|}{ Allocation } \\
\hline Sequences & 0 & 0 & 1 & 1 & 1 & 1 & 1 & 1 & 1 & 1 & 1 & 1 \\
\hline \multicolumn{13}{|l|}{ Allocation } \\
\hline Concealment & 0 & 0 & 0 & 0 & 0 & 0 & 0 & 0 & 0 & 0 & 0 & 0 \\
\hline \multicolumn{13}{|l|}{ Detailed } \\
\hline Intervention & 0 & 0 & 1 & 1 & 1 & 1 & 1 & 1 & 1 & 1 & 1 & 1 \\
\hline $\begin{array}{l}\text { Intervention } \\
\text { had appropriate } \\
\text { skill }\end{array}$ & 1 & 1 & 1 & 1 & 1 & 1 & 1 & 1 & 1 & 1 & 1 & 1 \\
\hline Adherence & 1 & 1 & 1 & 1 & 1 & 1 & 1 & 1 & 1 & 1 & 1 & 1 \\
\hline \multicolumn{13}{|l|}{ Blinding- } \\
\hline Participants & .5 & .5 & .5 & .5 & .5 & .5 & .5 & .5 & .5 & .5 & .5 & .5 \\
\hline \multicolumn{13}{|l|}{ Blinding-- } \\
\hline Interventionist & .5 & .5 & .5 & .5 & .5 & .5 & .5 & .5 & .5 & .5 & .5 & .5 \\
\hline \multicolumn{13}{|l|}{ Blinding- } \\
\hline Assessors & 1 & 1 & 1 & 1 & 1 & 1 & 1 & 1 & 1 & 1 & 1 & 1 \\
\hline \multicolumn{13}{|l|}{ Follow-Up } \\
\hline Schedule & 1 & 0 & 0 & 0 & 0 & 1 & 0 & 0 & 0 & 0 & 0 & 0 \\
\hline $\begin{array}{l}\text { Intention to } \\
\text { Treat }\end{array}$ & 0 & 0 & 0 & 0 & 0 & 0 & 0 & 0 & 0 & 0 & 0 & 0 \\
\hline Total Score & 5 & 4 & 6 & 6 & 6 & 7 & 6 & 6 & 6 & 6 & 6 & 6 \\
\hline
\end{tabular}

\section{Results}

The database and reference list searches yielded $142^{20}$ articles/book chapters. All articles were screened first by title ${ }^{21}$ which resulted in 25 duplicate articles being removed. The 22 abstracts of the remaining 117 articles were reviewed. $81^{23}$ articles were excluded as they were book chapters, clinical 24 reports, or the interventionist was not a music therapists. The 25 remaining 36 full-text articles were assessed for eligibility. $24^{26}$ articles were excluded based on this review: 23 articles were 27 not randomized controlled trials and 1 article included both 28 adolescents and adults. The remaining 12 studies are included 29 in this review

\section{Study Descriptions}

A summary of the studies included in this review may be found in Table 3 (participant characteristics) and Table 4 (study design information). All the studies were conducted in inpatient substance abuse treatment facilities, 9 of those with patients in detoxification. Sample sizes ranged from 16 to 141 with a combined total of 1124 individuals (544 females; 598 males; 9 did not identify gender) participating in studies designed to test the effects of receptive, recreative and compositional [10] methods on physiological and psychological effects of substance dependence. The majority of studies (9) included in this review were single-session music therapy session on short term detoxification units completed by the same researcher. 
1

2

\begin{tabular}{|c|c|c|c|c|}
\hline Author & $\begin{array}{c}\# \text { of } \\
\text { Participants } \\
E=9\end{array}$ & Gender & $\begin{array}{c}\text { Age } \\
\text { Range }\end{array}$ & $\begin{array}{l}\text { \# of } \\
\text { Sessions }\end{array}$ \\
\hline $\begin{array}{l}\text { Hammer } \\
(1996)\end{array}$ & $\begin{array}{c}\mathrm{C}-7 \\
\mathrm{~T}=16\end{array}$ & $\begin{array}{l}F=7 \\
M=9\end{array}$ & $26-61$ & 10 \\
\hline $\begin{array}{l}\text { Heiderscheit } \\
(2006)\end{array}$ & $\begin{array}{c}E=10 \\
C=9 \\
T=19\end{array}$ & $\begin{aligned} \mathrm{F} & =4 \\
\mathrm{M} & =15\end{aligned}$ & $42-64$ & $\begin{array}{c}\text { Max of } \\
7\end{array}$ \\
\hline $\begin{array}{l}\text { Murphy } \\
\text { (2009) }\end{array}$ & $\begin{array}{c}E=9 \\
C=7 \\
T=16\end{array}$ & $\begin{array}{l}F=7 \\
M=9\end{array}$ & $19-55$ & 8 \\
\hline $\begin{array}{l}\text { Silverman } \\
(2009)\end{array}$ & $\begin{array}{l}\mathrm{E}=29 \\
\mathrm{C}=37 \\
\mathrm{~T}=66\end{array}$ & $\begin{array}{c}F=36 \\
M=29 \\
\text { No } \\
\text { response } \\
=1\end{array}$ & $28-53$ & 1 \\
\hline $\begin{array}{l}\text { Silverman } \\
(2010)\end{array}$ & $\begin{array}{c}\mathrm{E}=64 \\
\mathrm{C}-54 \\
\mathrm{~T}=118\end{array}$ & $\begin{array}{c}F=56 \\
M=57 \\
\text { No } \\
\text { response } \\
=5\end{array}$ & $25-53$ & 1 \\
\hline $\begin{array}{l}\text { Silverman } \\
\text { (2011a) }\end{array}$ & $\begin{array}{c}\mathrm{E}=69 \\
\mathrm{C}=71 \\
\mathrm{~T}=140\end{array}$ & $\begin{array}{l}\mathrm{F}=70 \\
\mathrm{M}=70\end{array}$ & $29-56$ & 1 \\
\hline
\end{tabular}

\begin{tabular}{|c|c|c|c|c|}
\hline $\begin{array}{l}\text { Silverman } \\
\text { (2011b) }\end{array}$ & $\begin{array}{c}\mathrm{GA}=42 \\
\mathrm{~GB}=43 \\
\mathrm{GC}=56 \\
\mathrm{~T}=141\end{array}$ & $\begin{array}{l}F=59 \\
M=82\end{array}$ & 24-51 & 1 \\
\hline $\begin{array}{l}\text { S } \\
\text { ilverman } \\
(2012)\end{array}$ & $\begin{array}{l}E=48 \\
C-51 \\
T=99\end{array}$ & $\begin{array}{c}F=51 \\
M=48\end{array}$ & $29-57$ & 1 \\
\hline $\begin{array}{l}\text { Silverman } \\
(2014)\end{array}$ & $\begin{array}{c}\mathrm{GA}=41 \\
\mathrm{~GB}=41 \\
\mathrm{C}=49 \\
\mathrm{~T}=131\end{array}$ & $\begin{array}{c}F=59 \\
M=70 \\
\text { No } \\
\text { response } \\
=2\end{array}$ & $20-55$ & 1 \\
\hline $\begin{array}{l}\text { Silverman } \\
(2015)\end{array}$ & $\begin{array}{c}\mathrm{E}=51 \\
\mathrm{C}=53 \\
\mathrm{~T}=104\end{array}$ & $\begin{array}{l}F=76 \\
M=57\end{array}$ & $28-54$ & 1 \\
\hline $\begin{array}{l}\text { Silverman } \\
\text { (2016a) }\end{array}$ & $\begin{array}{c}\mathrm{E}=60 \\
\mathrm{C}=84 \\
\mathrm{~T}=144\end{array}$ & $\begin{array}{l}F=64 \\
M=79\end{array}$ & $23-55$ & 1 \\
\hline $\begin{array}{l}\text { Silverman } \\
\text { (2016b) }\end{array}$ & $\begin{array}{l}\mathrm{GA}=37 \\
\mathrm{~GB}=30 \\
\mathrm{GC}=29 \\
\mathrm{GD}=33 \\
\mathrm{~T}=129\end{array}$ & $\begin{array}{c}\mathrm{F}=55 \\
\mathrm{M}=73 \\
\text { No } \\
\text { response } \\
-2\end{array}$ & $22-55$ & 1 \\
\hline
\end{tabular}

3

Table 4: Music Therapy Method, Outcome Measure \& Result

\begin{tabular}{|c|c|c|c|c|c|}
\hline Author & Design & Music Therapy Method & $\begin{array}{l}\text { Dependent } \\
\text { Variable }\end{array}$ & Outcome Measures & Results \\
\hline Hammer (1996) & Pretest/posttest RCT & $\begin{array}{l}\text { EC: Guided imagery and } \\
\text { music } \\
\text { CC: } \text { Standard care }\end{array}$ & Anxiety & $\begin{array}{l}\text { State-Trait Anxiety } \\
\text { Inventory (STAI) }\end{array}$ & $\begin{array}{l}\text { Significant decrease in } \\
\text { state anxiety }\end{array}$ \\
\hline \multirow[t]{3}{*}{$\begin{array}{l}\text { Heiderscheit } \\
(2006)\end{array}$} & \multirow[t]{3}{*}{$\begin{array}{l}\text { Quasi-experimental } \\
\text { pretest/posttest }\end{array}$} & $\begin{array}{l}\text { EC: Individual BMGIM } \\
\text { CC: Standard care }\end{array}$ & $\begin{array}{l}\text { Interpersonal } \\
\text { Problems }\end{array}$ & $\begin{array}{l}\text { Interpersonal Problems } \\
\text { Short Circumplex Form } \\
\text { (IIP-SC) }\end{array}$ & \multirow[t]{3}{*}{$\begin{array}{l}\text { Significant decreases on } \\
\text { the domineering, cold } \\
\text { and non-assertive } \\
\text { subscales of the IIP-SC } \\
\text { \& on the manageability } \\
\text { subscale of the SOC }\end{array}$} \\
\hline & & & Salivary IgA & Saliva Samples & \\
\hline & & & Depression & Beck Depression Inventory & \\
\hline \multirow{2}{*}{ Murphy (2009) } & \multirow{2}{*}{ Pretest/posttest RCT } & $\begin{array}{c}\text { EC: Bonny Method of } \\
\text { Group guided }\end{array}$ & Sense of Coherence & $\begin{array}{l}\text { Sense of Coherence } \\
\text { (Antonovsky) }\end{array}$ & \multirow{2}{*}{ No significant results } \\
\hline & & $\begin{array}{l}\text { 1magery with music } \\
\text { CC: Standard care }\end{array}$ & $\begin{array}{l}\text { Motivation for } \\
\text { Treatment }\end{array}$ & $\begin{array}{l}\text { Importance, Confidence, } \\
\text { Readiness Ruler* }\end{array}$ & \\
\hline
\end{tabular}




\begin{tabular}{|c|c|c|c|c|c|}
\hline \multirow[t]{2}{*}{ Silverman (2009) } & \multirow[t]{2}{*}{$\begin{array}{l}\text { Cluster randomized 2- } \\
\text { group posttest only }\end{array}$} & \multirow[t]{2}{*}{$\begin{array}{l}\text { EC: Lyric analysis } \\
\text { CC: Verbal therapy }\end{array}$} & $\begin{array}{l}\text { Treatment } \\
\text { Eagerness }\end{array}$ & $\begin{array}{l}\text { Stages of Change Readiness } \\
\text { \& Treatment Eagerness } \\
\text { Scale (SOCRATES) version } \\
8 \mathrm{D}\end{array}$ & \multirow[t]{2}{*}{ No significant results } \\
\hline & & & Working Alliance & $\begin{array}{l}\text { Helping Alliance } \\
\text { Questionnaire (HAQ-III) }\end{array}$ & \\
\hline \multirow[b]{2}{*}{ Silverman (2010) } & \multirow[b]{2}{*}{$\begin{array}{l}\text { Randomized two } \\
\text { group posttest only }\end{array}$} & \multirow[b]{2}{*}{$\begin{array}{l}\text { EC: Lyric analysis } \\
\text { CC: Verbal therapy }\end{array}$} & $\begin{array}{l}\text { Withdrawal } \\
\text { Symptoms }\end{array}$ & $\begin{array}{l}\text { Adjective Rating Scale for } \\
\text { Withdrawal }^{*}\end{array}$ & \\
\hline & & & Locus of control & $\begin{array}{l}\text { Drinking-Related Internal- } \\
\text { External Locus of Control } \\
\text { Scale }\end{array}$ & No significant results \\
\hline \multirow[t]{2}{*}{$\begin{array}{l}\text { Silverman } \\
\text { (2011a) }\end{array}$} & \multirow[t]{2}{*}{$\begin{array}{l}\text { Randomized two } \\
\text { group posttest \& } \\
\text { follow-up }\end{array}$} & \multirow[t]{2}{*}{$\begin{array}{l}\text { EC: Blues songwriting } \\
\text { CC: Verbal therapy }\end{array}$} & $\begin{array}{l}\text { Motivation for } \\
\text { Change }\end{array}$ & $\begin{array}{l}\text { University of Rhode Island } \\
\text { Change Assessment } \\
\text { (URICA) }\end{array}$ & $\begin{array}{l}\text { Significantly higher } \\
\text { perceptions of } \\
\text { helpfulness, enjoyment, } \\
\text { and comfort } \\
\text { 1month follow-up: No }\end{array}$ \\
\hline & & & Depression & $\begin{array}{l}\text { Beck Depression Inventory } \\
\text { (Second Ed.) }\end{array}$ & Significant difference \\
\hline $\begin{array}{l}\text { Silverman } \\
(2011 b)\end{array}$ & $\begin{array}{l}\text { Cluster randomized } \\
\text { three group posttest } \\
\text { only }\end{array}$ & $\begin{array}{l}\text { GA Rockumentary } \\
\text { GB: Verbal Therapy } \\
\text { GC: Recreational MT }\end{array}$ & $\begin{array}{l}\text { Readiness to } \\
\text { Change }\end{array}$ & $\begin{array}{l}\text { Readiness to Change } \\
\text { Questionnaire- } \\
\text { Treatment Version }\end{array}$ & $\begin{array}{l}\text { GA \& GC had } \\
\text { significantly higher } \\
\text { scores for } \\
\text { Contemplation \& } \\
\text { Action }\end{array}$ \\
\hline & & & $\begin{array}{l}\text { Cravings } \\
\text { Motivation \& } \\
\text { Readiness for }\end{array}$ & $\begin{array}{l}\text { Scale }^{\star} \\
\text { Circumstance, Motivation, } \\
\text { \& Readiness Scales for }\end{array}$ & $\begin{array}{l}\text { Songwriting condition } \\
\text { Significant results for }\end{array}$ \\
\hline Silverman (2012) & $\begin{array}{l}\text { Cluster randomized } \\
\text { posttest only }\end{array}$ & $\begin{array}{l}\text { EC: Blues songwriting } \\
\text { CC: Wait-list }\end{array}$ & Treatment & $\begin{array}{l}\text { Substance Abuse } \\
\text { Treatment }\end{array}$ & $\begin{array}{l}\text { motivation \& } \\
\text { readiness for } \\
\text { treatment }\end{array}$ \\
\hline Silverman (2014) & $\begin{array}{l}\text { Cluster randomized } \\
\text { three group posttest } \\
\text { only }\end{array}$ & $\begin{array}{l}\text { GA: Lyric analysis } \\
\text { GB: Verbal therapy } \\
\text { GC: Wait-list }\end{array}$ & $\begin{array}{l}\text { Self-efficacy for } \\
\text { avoiding addictive } \\
\text { behaviors }\end{array}$ & $\begin{array}{l}\text { Drug Avoidance Self- } \\
\text { Efficacy Scale }\end{array}$ & $\begin{array}{l}\text { No significant } \\
\text { differences }\end{array}$ \\
\hline Silverman (2015) & $\begin{array}{l}\text { Cluster randomized } \\
\text { posttest only }\end{array}$ & $\begin{array}{l}\text { EC: Lyric analysis } \\
\text { CC: Wait-list }\end{array}$ & $\begin{array}{l}\text { Treatment } \\
\text { Motivation }\end{array}$ & $\begin{array}{l}\text { Texas Christian University } \\
\text { Treatment Motivation } \\
\text { Scale-Client Evaluation } \\
\text { of Self at Intake (CESI) }\end{array}$ & $\begin{array}{l}\text { Lyric analysis group } \\
\text { had significant } \\
\text { differences in } \\
\text { measures of problem } \\
\text { recognition, desire for } \\
\text { help, treatment } \\
\text { readiness, and total } \\
\text { motivation }\end{array}$ \\
\hline Silverman & $\begin{array}{l}\text { Group based cluster } \\
\text { randomized single }\end{array}$ & EC: Lyric analysis & $\begin{array}{l}\text { Withdrawal } \\
\text { Symptoms }\end{array}$ & $\begin{array}{l}\text { Adjective Rating Scale for } \\
\text { Withdrawal }^{*} \text { (ARSW) }\end{array}$ & $\begin{array}{l}\text { No significant } \\
\text { differences }\end{array}$ \\
\hline (2016a) & session wait-list & CC: Wait-list & Cravings & $\begin{array}{l}\text { Brief Substance Craving } \\
\text { Scale* (BSCS) }^{*}\end{array}$ & \\
\hline $\begin{array}{l}\text { Silverman } \\
(2016 \mathrm{~b})\end{array}$ & $\begin{array}{l}4 \text { Group cluster- } \\
\text { randomized design }\end{array}$ & $\begin{array}{l}\text { EC1: Live educational MT } \\
\text { EC2: Recorded educational } \\
\text { MT } \\
\text { EC3: Education w/o music } \\
\text { EC4: Recreational MT }\end{array}$ & Working Alliance & $\begin{array}{l}\text { Working Alliance } \\
\text { Inventory-Short Form } \\
\text { (WAI-S) } \\
\text { Wake Forest Physician } \\
\text { Trust Scale }\end{array}$ & $\begin{array}{l}\text { No significant } \\
\text { differences }\end{array}$ \\
\hline
\end{tabular}




\section{Quality Assessment}

The studies included in this review received an average quality score of 5.8 (range 4 to 6 ; mode $=6$; median $=6$ ). Based on these scores all studies were assumed to have a moderate risk of bias. Areas where studies did not meet criteria included allocation concealment, and use of intention-to-treat procedures. Only 2 studies $[11,12]$ included post-study follow-up in the study design. See Table 2[13] for Quality Assessment Scores.

\section{Intervention Reporting}

10 of the 12 studies reviewed meet the intervention reporting guidelines (see Table 1 for intervention reporting criteria which is based on Robb et al.). In order to meet the intervention reporting guidelines, authors must have included 1) person selecting the music; 2) type of music selected and references for recorded/sheet music); 3) music delivery method; 4) materials; 5) intervention strategies and 6) intervention delivery schedule. Lack of information on the type of music and references was the most common reason to determine a study did not meet intervention reporting criteria.

\section{Music Therapy Methods and Outcomes}

Three studies compared music and imagery methods with standard care $(11,14,15) ; 2$ studies compared lyric analysis with verbal therapy $[16,17]$ or used a wait-list control group $[18,19]$. Two studies compared songwriting to verbal therapy $[12,20]$. One study compared 2 music therapy methods (rockumentary vs. recreational music therapy) to verbal therapy [21] and one compared lyric analysis with a no-music relapse prevention group and a wait-list control group [22]. Finally, one study compared 4 treatment conditions: live educational music therapy, recorded educational music therapy, education without music and recreational music [23]. Definitions for all music therapy methods may be found in Appendix A.

Dependent variables included anxiety [11]; interpersonal problems [14]; sense of coherence [14,15]; salivary Immunoglobulin A (Salivary IgA) [14]; depression (12, 15]; readiness/motivation to change $[12,15,16,18,20,21]$; working alliance [16, 23]; withdrawal symptoms [17, 23]; locus of control [17] cravings [19, 21] and drug-avoidance self-efficacy [22].

Studies involving music and imagery had mixed results. State anxiety as measured by the STAI was significantly decreased after 10 weekly guided imagery through music sessions [11] Scores on the domineering, cold and nonassertive subscale of the IIP-SC and the manageability subscale of the SOC were significantly decreased after a series of 7 to 10 individual Bonny Method of Guided Imagery and Music (BMGIM) sessions; however, salivary IgA levels were not impacted [14]. Significant results were not obtained on, depression as measured by the BDI, sense of coherence as measured by the SOC, or motivation as measured by the ICR after a series of 8 group guided imagery and music sessions [15].

Studies involving lyric analysis also had mixed results. Participants in the music therapy group scored significantly higher on the problem recognition, desire for help, treatment readiness, and motivation subscales of the CESI than those in the wait-list control group [18]. Participants in both the "rockumentary" group and the recreational music therapy group had significantly higher scores on the Contemplation and Action subscales of the RCQ-TV than those in the verbal therapy group [21]. Significant results were not found for treatment eagerness as measured by the SOCRATES [16] working alliance as measured by the WAI-S [16, 23], withdrawal symptoms as measured by the ARSW [17, 23], locus of control as measured by the DRIE [17] depression as measured by the BDI-II [12], readiness to change as measured by the RCQ-TV [21], or cravings as measured by the BSCS [21].

Studies examining the efficacy of songwriting also had mixed results. In one study, blues songwriting was found to significantly increase scores on the motivation and readiness for treatment subscales of the CMR [20]. Blues songwriting was also found to significantly improve treatment perception on a therapist designed Likert scale, however, it had no effect on depression as measured by the BDI-II or motivation for change as measured by the URICA [12]

\section{Conclusions \& Recommendations}

This review set out to summarize the current evidence which would support the inclusion of music therapy as a primary treatment modality in addictions treatment. Research fitting the inclusion criteria studied the efficacy of music and imagery, lyric analysis, songwriting, and recreational music therapy. The findings of this review are mixed. In some cases, each of these methods was found to have significant results; other times results were not significant.

All studies included in this review had a moderate to high risk of bias. The following recommendations are made to help researchers reduce the risk of bias: 1) blind researchers, (interventionists) and participants to treatment allocation until after consent has been obtained; 2) follow intervention reporting guidelines as recommended by Robb et al. for all music-based interventions; 3 ) outcome measures and analysis should be performed by someone other than the researcher (interventionist); and 4) follow intention-to-treat guidelines.

Future studies should investigate the inclusion of music therapy in a variety of treatment settings, both inpatient and outpatient; group and individual. The majority of studies included in this review conducted on an inpatient detoxification unit using a single-session posttest only design. 
Studies in longer term settings may yield significant results on the outcomes studied in the single session format.

The studies included in this review investigated a limited number of music therapy methods. The clinical literature reports on the use of a wider variety of music therapy methods and outcomes $[24,25,26]$. These reports can lay the groundwork for future investigations on both methods and outcomes.

\section{Limitations}

This study only examined published research studies that were indexed in PsycInfo, Medline, and CINAHL databases, or found in the reference lists of articles identified through the search. Master's thesis, dissertations and unpublished studies were not included. Additionally, only studies published in English were included. Lastly, qualitative research studies were not included. Historically, systematic reviews have evaluated experimental research (typically RCTs). However, recently there has been a shift and qualitative studies have been included [27] In the future, researchers may want to broaden the scope of their review to include those types of studies not reviewed for this report.

Clearly, the jury is out and a formal recommendation for or against the inclusion of music therapy as a primary treatment modality in addictions treatment would be premature. The findings of this review support continued research in order to ascertain the relationship between music therapy methods and clinical outcomes. Future studies, with a low risk of bias, and high quality intervention reporting are needed in order to support the inclusion of music therapy in addictions treatment.

\section{References}

1. Substance Abuse and Mental Health Services Administration, Center for Behavioral Health Statistics and Quality. Treatment Episode Data Set (TEDS): 2004-2014. National Admissions to Substance Abuse Treatment Services. BHSIS Series S-84, HHS Publication No. (SMA) 16-4986. Rockville, MD: Substance Abuse and Mental Health Services Administration, 2016.

2. Aletraris L, Paino M, Edmond MB, Poman PM. The use of art and music therapy in substance abuse treatment programs. J Addict Nurs. 2014; 25:190-195. doi:10.1097/jan000000000000048

3. Higgins JPT, Green S. (eds). Cochrane Handbook for Systematic Reviews of Interventions Version 5.1.0 [updated March 2011]. The Cochrane Collaboration, 2011. Available from www.handbook.cochrane.org.

4. McKinney $\mathrm{CH} \&$ Honig TJ. Health outocmes of a series of Bonny method of guided imagery and music sessions: A systematic review. $J$ Mus Ther. 2016; Advance online access:1-34. doi:10.1093/jmtthw016

5. Hanson-Abromeit D \& Moore KS. The systematic review as a research process in music therapy. J Mus Ther. 2014; 51:4-38. doi:10.1093/jmt/thu002
6. Mays KL, Clark, DL, Gordon AJ. Treating addiction with tunes: A systematic review of music therapy for the treatment of patients with addictions. Subst Abus. 2008; 29:51-59.

7. Moher D, Liberati A, Tetzlaff J, Altman DG, The PRISMA Group. Preferred reporting items for systematic reviews and meta-analyses: The PRISMA statement. PLoS Med 6(6) e1000097. doi:10.1371/journal.pmed1000097. Retrieved from www.prismnsstatement.org

8. Boutron L, Moher D, Tugwell P, Giraudeau B, Poiraudeau S, Nizard R, et al. A checklist to evaluate a report of nonpharmacological trial (CLEAR NPT) was developed using consensus. J Clin Epidemiol. 2005; 58: 1233-40. doi:10.1016/j.jclinepi.2005.05.004

9. Yinger OS, Gooding LF. A systematic review of music-based interventions for procedural support. J Music Ther. 2015; 52: 1-77. doi:10.1093/jmt/thv004

10. Bruscia KE. Defining Music Therapy ( $3^{\text {rd }}$ ed). Gilsum, NH: Barcelona Publishers, 2014

11. Hammer SE. The effects of guided imagery through music on state and trait anxiety. J Music Ther. 1996; 33: 47-40.

12. Silverman MJ. Effects of Effects of music therapy on change and depression on clients in detoxification. J Addict Nurs. 2011; 22:185-92.

13. Robb SL, Burns DS. \& Carpenter JS. Reporting guidelines for musicbased interventions. Music Med; 2011: 3 271-79. doi: $10.1177 / 1943862111420539$

14. Heiderscheit A. The effects of the Bonny Method of Guided Imagery and Music on interpersonal problems, sense of coherence and salivary immunoglobulin a of adults in chemical dependency treatment. Dissertation Abstracts International, 66, 2006.

15. Murphy KM. The effects of group guided imagery and music on the psychological health of adults in substance abuse treatment. Dissertation Abstracts International Section A: Humanities and Social Sciences, 69(8-A), pp. 2939. 2009

16. Silverman MJ. The effect of lyric analysis on treatment eagerness and working alliance in consumers who are in detoxification: A randomized clinical effectiveness study. Music Ther Perspec, 2009; 27: 115-21.

17. Silverman MJ. The effect of a lyric analysis intervention on withdrawal symptoms and locus of control in patients on a detoxification unit: A randomized effectiveness study. Arts Psychother. 2010; 37: 197-201.

18. Silverman MJ. Effects of lyric analysis interventions on treatment motivation in patients on a detoxification unit: A randomized effectiveness study. J Music Ther. 2015; 52:117-34. doi:10.1093/jmt/thu057

19. Silverman MJ. Effects of a single lyric analysis intervention on withdrawal and craving with inpatients on a detoxification unit: A cluster-randomized effectiveness study. Subst Use Misuse. 2016; 51: 24149. doi:10.3109/10826084.2015.1092990

20. Silverman MJ. Effects of group songwriting on motivation and readiness for treatment on patients in detoxification: A randomized wait-list effectiveness study. J Music Ther. 2012; 49: 414-29. doi:10.1093/jmt/49.4.414

21. Silverman MJ. Effects of music therapy on change readiness and craving in patients on a detoxification unit. J Music Ther. 2011; 48:509-31.

22. Silverman MJ. Effects of music therapy on drug avoidance self-efficacy in patients on a detoxification unit: A three-group randomized effectiveness study. $J$ Addict Nurs. 2014; 25: 172-81. doi:10.1097/JAN.0000000000000047

23. Silverman MJ. Effects of Live and Educational Music Therapy on Working Alliance and Trust With Patients on Detoxification Unit: A Four-Group Cluster-Randomized Trial. Subst Use Misuse. 2016; 51: 1741-50. doi:10.1080/10826084.2016.1197263

24. Ficken, T. Music therapy with chemically dependent clients: A relapse prevention model. In Aldridge D \& Fachner J, Eds. Music therapy and addictions. Philadelphia, PA: Jessica Kingsley Publishers; 2010:103-112.

25. Gardstrom SC, Bartkowski, J, Willenbrink J, \& Diestelkamp WS. The impact of group music therapy on negative affect of people with co- 
occurring substance use disorders and mental illnesses. Music Ther Perspec; 31: 116-126

26. Treder-Wolff J. Affecting attitudes: Music therapy in addictions treatment. Music Ther Perspect. 1990; 8: 67-71. doi:10.1093/mtp/8.1.67

27. Denny E. Reviewing qualitative evidence to evaluate patient experience. In: Khan K, Junz R, Kleijnen J, \& Antes G, Eds. Systematic reviews to support evidence-based medicine. $2^{\text {nd }}$ ed. London, England: Hodder \& Stoughton; 2011: 585-606.

28. Bruscia KE. Defining music therapy $3^{\text {rd }}$ ed.. Gilsum, NH: Barcelona Publishers. 2014.

\section{Biographical Statements}

Kathleen M. Murphy, PhD, MT-BC is the Coordinator of the Music Therapy Program at Loyola University. In addition to teaching she is an active researcher and maintains a small private practice. 